Article

\title{
Intersubband Transition Engineering in the Conduction Band of Asymmetric Coupled Ge/SiGe Quantum Wells
}

\author{
Luca Persichetti ${ }^{1}{ }^{\circledR}$, Michele Montanari ${ }^{1}{ }^{\circledR}$, Chiara Ciano $^{1}$, Luciana Di Gaspare ${ }^{1}$, \\ Michele Ortolani ${ }^{2}{ }^{\mathbb{D}}$, Leonetta Baldassarre ${ }^{2}$, Marvin Zoellner ${ }^{3}$, Samik Mukherjee ${ }^{4}$, \\ Oussama Moutanabbir ${ }^{4}$, Giovanni Capellini ${ }^{1,3}$ (-) , Michele Virgilio ${ }^{5}$ and Monica De Seta ${ }^{1, *}$ \\ 1 Department of Sciences, Università Roma Tre, Viale G. Marconi 446, I-00146 Rome, Italy; \\ luca.persichetti@uniroma3.it (L.P.); michele.montanari@uniroma3.it (M.M.); chiara.ciano@uniroma3.it (C.C.); \\ luciana.digaspare@uniroma3.it (L.D.G.); capellini@ihp-microelectronics.com (G.C.) \\ 2 Department of Physics, Sapienza University of Rome, Piazzale Aldo Moro, I-00185 Rome, Italy; \\ michele.ortolani@roma1.infn.it (M.O.); leonetta.baldassarre@roma1.infn.it (L.B.) \\ 3 IHP-Leibniz-Institut für innovative Mikroelektronik, Im Technologiepark 25, D-15236 Frankfurt (Oder), \\ Germany; zoellner@ihp-microelectronics.com \\ 4 Department of Engineering Physics, École Polytechnique de Montréal, C. P. 6079, Succ. Centre-Ville, \\ Montréal, QC H3C 3A7, Canada; samik.mukherjee@polymtl.ca (S.M.); \\ oussama.moutanabbir@polymtl.ca (O.M.) \\ 5 Department of Physics “Enrico Fermi”, Università di Pisa, Largo Pontecorvo 3, I-56127 Pisa, Italy; \\ michele.virgilio@unipi.it \\ * Correspondence: monica.deseta@uniroma3.it; Tel.: +39-06-5733-3430
}

Received: 1 February 2020; Accepted: 4 March 2020; Published: 6 March 2020

\begin{abstract}
Ge/SiGe asymmetric coupled quantum wells represent the building block of a variety of nanoscale quantum devices, including recently proposed designs for a silicon-based $\mathrm{THz}$ quantum cascade laser. In this paper, we combine structural and spectroscopic experiments on 20-module superstructures, each featuring two Ge wells coupled through a Ge-rich SiGe tunnel barrier, as a function of the geometry parameters of the design and the P dopant concentration. Through a comparison of $\mathrm{THz}$ spectroscopic data with numerical calculations of intersubband optical absorption resonances, we demonstrated that it is possible to tune, by design, the energy and the spatial overlap of quantum confined subbands in the conduction band of the heterostructures. The high structural/interface quality of the samples and the control achieved on subband hybridization are promising starting points towards a working electrically pumped light-emitting device.
\end{abstract}

Keywords: quantum wells; group IV epitaxy; intersubband transitions; silicon-germanium heterostructures; $\mathrm{THz}$ spectroscopy

\section{Introduction}

Semiconductor multi-quantum wells (MQWs) represent the perfect playroom for nanoscale scientists to devise novel technologies and device architectures using the fundamental laws of quantum mechanics [1,2]. In the last decades, the number of applications based on a MQW structure core has been constantly expanding, and now ranges from photovoltaics and solar energy harvesting [3-5] to photonics [6-8] and spin-based electronics [9,10]. Such a great technological potential exploits the tunability of the MQW design for precisely controlling the wavelength of electromagnetic radiation emitted or absorbed in the transitions between quantized states of the heterostructure. Compared to interband light emitters, unipolar devices based on intersubband (ISB) transitions in the conduction 
or valence band of the QW, such as quantum cascade lasers (QCLs) [11] and quantum fountains [12], offer a straightforward route to access the $\mathrm{THz}$ frequency domain and to extend the range of viable light-emitting materials to non-direct bandgap semiconductors $[13,14]$. As a matter of fact, since in these unipolar devices the radiative decay rate does not depend on electron-hole recombination, efficient light emitters can be envisaged with group IV materials, such as Ge, SiGe alloys [14-16] and GeSn $[17,18]$, which can be monolithically grown on silicon wafers and, thus, integrated in the complementary metal-oxide-semiconductor (CMOS) platform. Electrically pumped QCLs and optically pumped quantum fountains realized in the SiGe material system also benefit from the absence of polar longitudinal optical phonons inducing the long-range polarization field (Fröhlich interaction) which strongly couples to charge carriers and limits their non-radiative lifetimes in III-V compounds [7]. Being only mediated by the short-range deformation potential, electron-phonon coupling in non-polar semiconductors is much weaker $[19,20]$ and, as a consequence, SiGe ISB lasers are expected to have a wider temperature operational range, reaching room temperature for $n$-type Ge/SiGe QCLs [21]. In addition, the emission bandwidth would widen, extending inside the reststrahlen band (5-10 THz) of forbidden light propagation caused, in III-V materials, by strong light absorption by optical phonons in this spectral range [7]. The most promising QCL design for successful lasing operation in the group IV material platform exploits ISB transitions of electrons in the conduction band $L$ valleys of Ge-rich heterostructures and features, as a building block, asymmetric coupled quantum wells (ACQWs) made of Ge layers of different thicknesses and coupled through $\mathrm{Si}_{1-x} \mathrm{Ge}_{x}$ barriers, typically with a Ge content $x=0.77 \div 0.85[22,23]$. QCL designs based on $n$-type Ge/SiGe heterostructures are predicted to enable peak gain values remarkably higher (more than $6 x$ at low temperature) than $p$-type emitters leveraging on hole-to-hole radiative transitions in Si-rich structures [13,14]. Due to the high Ge content, the epitaxial integration of such Ge-rich structures on Si wafers nonetheless represents a challenging task, with still open issues ranging from growth optimization, i.e., the management of strain relaxation for obtaining structural and interface quality with atomic-scale control of composition profiles, to the need for an accurate determination of band offsets from $\mathrm{THz}$ spectroscopy experiments. To address these issues, Ge/SiGe ACQWs represent an ideal model system which inherently presents some of the main challenges for a future QCL development in the SiGe system [21], such as (i) achieving high structural and interface quality in strain-compensated Ge-rich heterostructures, despite their large lattice mismatch with the Si substrate; (ii) controlling the concentration profile of $n$-type dopant $P$ atoms during the epitaxial growth and quantification of the profile broadening due to the surface segregation of donors; (iii) designing the energy of ISB transitions and the engineering of electron wavefunctions with the desired degree of delocalization.

As a necessary milestone on the way towards a SiGe based QCL, we investigate in this paper n-doped $\mathrm{Ge} / \mathrm{Si}_{0.20} \mathrm{Ge}_{0.80} \mathrm{ACQWs}$ grown by chemical vapor deposition (CVD), combining structural analysis techniques with $\mathrm{THz}$ spectroscopy as a probe of interwell coupling and level broadening due to interface roughness and ionized impurity scatterings. By modulating the heterostructure design (i.e., tunnel barrier thickness and relative width of the two QWs), we explore a wide range of ISB energies (different alignment of quantum-confined levels) and spatial distribution of the wavefunctions corresponding to excited confined states, obtaining a consistent match of the experimental data to numerical calculations performed through a multivalley effective mass model based on a Schrödinger-Poisson solver. This systematic study allows us to accurately determine material parameters for the SiGe system, such as interface broadenings and L-point conduction band offsets, which are mandatory to establish a reliable simulation platform for QCL optimization. 


\section{Materials and Methods}

Strain-compensated MQW heterostructures were grown by ultra-high vacuum CVD at $500{ }^{\circ} \mathrm{C}$ using ultrapure germane and silane without carrier gases. The reacting gas pressure was $1.2 \mathrm{mTorr}$, at which the typical growth rate was $6.5 \mathrm{~nm} / \mathrm{min}$ for $\mathrm{Ge}$ and $4.5 \mathrm{~nm} / \mathrm{min}$ for a $\mathrm{Si}_{0.20} \mathrm{Ge}_{0.80}$ alloy. The sample design (sketched in Figure 3c) featured a wide and a narrow Ge well of thickness $w_{L}$ and $w_{t}$, respectively. The wells were separated by a SiGe tunnel barrier of thickness $b_{t}$ and Ge composition $x_{G e}$. Among the different samples, $w_{t}=5.0 \mathrm{~nm}$ was kept constant, $w_{L}$ was varied between 11.3 and $16.0 \mathrm{~nm}$ and $b_{t}$ ranged between 2.3 and $5 \mathrm{~nm}$. The value of $x_{G e}$ was set to 0.81 or 0.87 , with the higher Ge composition describing the more pronounced SiGe intermixing for $b_{t}<3 \mathrm{~nm}$ [21]. The wide well was $n$-doped by phosphine co-deposition over a thickness $t=10 \mathrm{~nm}$. In the samples, the module, composed by the two Ge wells and the SiGe barrier separating them, was been repeated 20 times with $21 \mathrm{~nm}$ thick $\mathrm{Si}_{0.20} \mathrm{Ge}_{0.80}$ spacers between the individual modules. Specific information on individual samples can be found in Table 1. The MQW stack was deposited on a relaxed $\mathrm{Si}_{0.15} \mathrm{Ge}_{0.85}$ alloy buffer with a thickness of $1.2 \mu \mathrm{m}$. In such high Ge content regime, low threading dislocation density (TDD $\sim 1 \times 10^{7}$ $\mathrm{cm}^{-2}$ ) was obtained through a reverse step-graded virtual substrate (RG-VS) [24]. In this approach, first, a plastically relaxed $700 \mathrm{~nm}$ thick Ge buffer was directly deposited on the $\mathrm{Si}(001)$ substrate and then, on top of it, two SiGe layers were deposited, each being $150 \mathrm{~nm}$ thick and with a Ge content, respectively of 0.95 and 0.90 (See schematics in Figure 1a). Structural characterization of the samples was performed by scanning transmission electron microscopy (STEM) using a FEI Titan microscope (FEI Company, Hillsboro, Oregon, USA), operated at $200 \mathrm{kV}$ and equipped with aberration-corrected magnetic lenses for obtaining electron probes of the order of 1-2 Å diameter with beam currents of 200 pA. A CEOS CESCOR corrector was used to yield a resolution of $0.8 \AA$. The images were recorded using a high-angle annular dark field (HAADF) detector (FEI Company, Hillsboro, Oregon, USA). The sample preparation for STEM was done in a dual channel focused ion beam (Dual-FIB) microscope, using the standard lamella lift-out technique. STEM measurements were coupled to high-resolution X-ray diffraction (XRD) analysis performed at room temperature with a Rigaku SmartLab tool with a rotating anode and line-focus geometry featuring a $\mathrm{Ge}(400) \times 2$ channel-cut beam collimator and a $\mathrm{Ge}(220) \times 2$ analyzer crystal. The typical broadening of spatial donor profiles due to the segregation and diffusion of $P$ atoms in our growth conditions was measured on calibration samples using dynamic secondary ion mass spectrometry (D-SIMS) on a CAMECA IMS Wf Tool, with oxygen at $400 \mathrm{eV}$ impact energy in positive mode, monitoring the $31 \mathrm{P}+$ signal. ISB absorption spectra were measured at $T=$ $10 \mathrm{~K}$ by means of Fourier transform infrared (FTIR) spectroscopy in a side-illuminated single-pass waveguide configuration with a Bruker Vertex $70 \mathrm{v}$ (Bruker, Billerica, Massachusetts, USA) equipped with a He-flow cryostat. The lateral facets of our $2.5 \mathrm{~mm}$ long samples were cut at a $70^{\circ}$ angle with respect to the growth plane and the top surface close to the MQWs was coated with a metal bilayer (Ti/Au $10 \mathrm{~nm} / 80 \mathrm{~nm}$ ) [25], in order to make the electric field of the radiation propagating through the MQWs almost parallel to the ISB dipole moment (i.e., TM polarized) [26]. The measured quantity was the dichroic transmission spectra $T(\omega)=T_{T M}(\omega) / T_{T E}(\omega)$, which ensured that polarization independent spectral features not related to ISB transitions (e.g. the dopant absorption in the Si wafer) were suppressed. From $T(\omega)$, we evaluated the dimensionless absorption coefficient $\alpha_{2 D}(\omega)$ and the sheet carrier density as in [27]. In the simulations, electron states and ISB absorption spectra were calculated self-consistently in a Schrödinger-Poisson iterative scheme with parabolic subband dispersion [27], in which depolarization shift effects, i.e., the blueshift of absorption peaks due to the screening of the radiation field by the collective plasma mode [25], were included. 
Table 1. Asymmetric coupled quantum well (ACQW) design. $b_{t}$ is the thickness of the tunnel barrier and $x_{G e}$ its composition. $w_{L}$ is the thickness of the wide well which is $n$-doped, except for sample 2223. For all the samples, the thin well is $5 \mathrm{~nm}$ thick, the ACQW module is repeated 20 times and the $\mathrm{Si}_{0.20} \mathrm{Ge}_{0.80}$ spacer thickness between the modules is $21 \mathrm{~nm}$. The multi-quantum well (MQW) periodicity $D^{S L}$ is measured from the spacing of the superlattice fringes in X-ray diffraction (XRD) rocking curves. Values in brackets are the nominal periodicities. The sheet carrier density $n_{2 D}$ is obtained from the Fourier transform infrared (FTIR) optical absorption spectra $\alpha_{2 \mathrm{D}}(\omega)$.

\begin{tabular}{|c|c|c|c|c|c|}
\hline Sample & $w_{L}(\mathrm{~nm})$ & $b_{t}(\mathrm{~nm})$ & $x_{G e}$ & $D^{S L}(\mathrm{~nm})$ & $n_{2 D}\left(\times 10^{11} \mathrm{~cm}^{-2}\right)$ \\
\hline 2216 & 12.0 & 2.3 & 0.87 & $41.6[40.3]$ & 7.2 \\
\hline 2217 & 13.0 & 2.3 & 0.87 & 42.8 [41.3] & 9.0 \\
\hline 2218 & 11.3 & 2.3 & 0.87 & $40.6[39.6]$ & 7.8 \\
\hline 2219 & 11.5 & 2.3 & 0.87 & $40.6[39.8]$ & 1.0 \\
\hline 2221 & 12.0 & 3.3 & 0.81 & $42.4[41.3]$ & 6.0 \\
\hline 2222 & 12.0 & 4.0 & 0.81 & $43.0[42.0]$ & 4.6 \\
\hline 2223 & 12.0 & 2.3 & 0.87 & 39.5 [40.3] & undoped \\
\hline 2224 & 12.0 & 5.0 & 0.81 & $43.9[43.0]$ & 1.5 \\
\hline 2267 & 16.0 & 2.3 & 0.81 & [44.3] & 3.0 \\
\hline
\end{tabular}
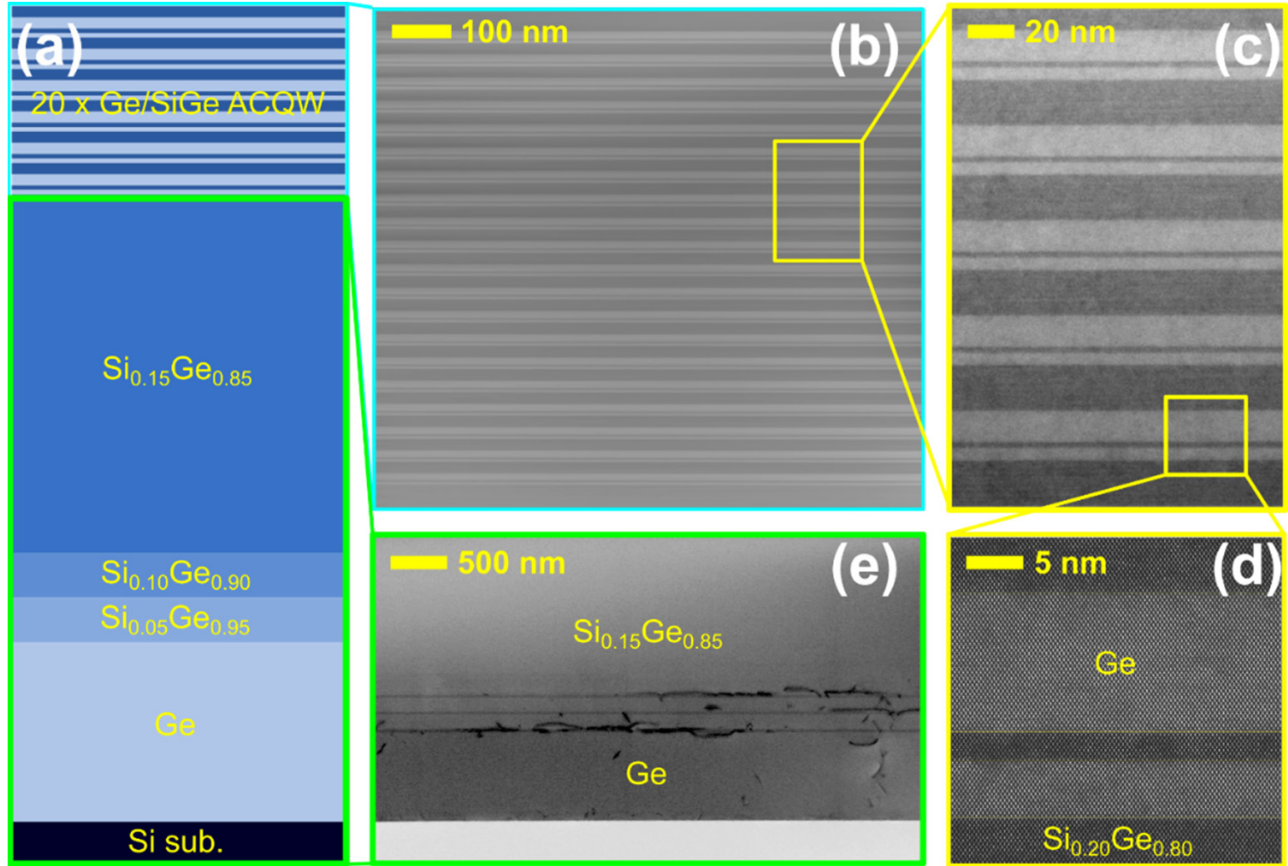

Figure 1. (a) Schematics of the structure of the grown heterolayers from the virtual substrate (VS) to the active region on top. (b-e) Scanning transmission electron microscopy (STEM) micrographs of a typical ACQW sample: panels (b-d) show increasing magnifications of the active region composed by the alternation of Ge wells and SiGe barriers at different compositions, as described in the text. Panel (e) reports the part of the VS underlying the active region being close to the Si substrate, including the Ge buffer and the reverse-step graded (RG) SiGe step layers.

\section{Results}

\subsection{Structural Characterization}

Figure 1 shows a full STEM characterization of a typical ACQW sample, probed at different length scales and depths along the VS and the overgrown MQW stack. Taking as a reference the sample structure sketched in Figure 1a, we fully characterize the grown stack, obtaining a high-resolution imaging of the active region composed by the 20 repeating ACQW modules (Figure 1b-d), as well as probing the RG-VS and the Ge buffer layer (Figure 1e) to characterize plastic relaxation at the bottom 
of the of $1.2 \mu \mathrm{m}$ thick $\mathrm{Si}_{0.15} \mathrm{Ge}_{0.85}$ alloy buffer. The images displayed in panels $\mathrm{c}$ and $\mathrm{d}$ show a zoom-up of the well/spacer $\left(\mathrm{Ge} / \mathrm{Si}_{0.20} \mathrm{Ge}_{0.80}\right)$ and the well/tunnel barrier $\left(\mathrm{Ge} / \mathrm{Si}_{0.13} \mathrm{Ge}_{0.87}\right)$ heterointerfaces, where atomic-resolved features are evident. Between the thick $\mathrm{Si}_{0.15} \mathrm{Ge}_{0.85}$ alloy and the Ge buffer in Figure $1 \mathrm{e}$, we clearly observe three interfaces corresponding to the 5\% steps in the Ge concentration decreasing from pure Ge to $\mathrm{Si}_{0.15} \mathrm{Ge}_{0.85}$. The STEM contrast is due to the misfit dislocations that pile up at each interface. Threading dislocation segments are only present in the relaxed Ge buffer and in the lower RG layers, whereas no threading dislocations are observed in the top $\mathrm{Si}_{0.15} \mathrm{Ge}_{0.85}$ alloy buffer. The absence of threading segments within the field of view of the image is consistent with the TDD count reported in the methods and makes visually clear the "filtering" effect produced by the heterointerfaces which hinder the propagation upward of threading dislocations [25]. At the origin, there is the effective gliding force due to the compositional change at the interface which drives the change of the character of dislocations from threading to misfit, as threading segments bend on the interface plane [28,29]. Focusing on the quality of the MQW stack, large-scale STEM images (an example is reported in Figure 1b) demonstrate the remarkably high reproducibility in the deposition process, resulting in a homogeneous periodicity along the growth direction. At high magnifications (Figure 1c,d), STEM shows sharp and abrupt interfaces, with a broadening due to SiGe intermixing in the order of $0.8 \mathrm{~nm}$ [21]. The quality of the heterointerfaces is confirmed by the low value of the root-mean-square interface roughness, which was estimated to be $0.18 \mathrm{~nm}$ by atomic probe tomography measurements performed on the same set of samples [30]. The thickness of wells and barriers reported in Table 1 was measured by energy-dispersive X-ray spectroscopy (EDX), while the periodicity of the MQW stack $D^{S L}$ was obtained by XRD. The latter provides the perfect tool for evaluating the structural quality and homogeneity at a length-scale of a few millimeters (i.e., the typical size of the X-ray beam), thus giving access to a spatial probe complementary to STEM.

Figure 2a shows a typical rocking curve, around the (004) Ge and (004) Si Bragg peaks, obtained on our ACQWs. As an exemplificative example, we report data for sample 2223. Together with the reflections due to the Ge buffer and the Si substrate, we observe the peak corresponding to the $\mathrm{Si}_{0.15} \mathrm{Ge}_{0.85}$ alloy buffer which is the thicker layer in the RG-VS. In addition, multiple orders of superlattice (SL) satellites emerge. Their high quality-factor confirms the high crystalline quality and sharpness of the MQW layers. A statistical analysis performed on the data reported in Table 1 for the entire set of samples reveals that the superlattice periodicity $D^{S L}$ matches the nominal values within $2.4 \%$ on average, with the maximum deviation, observed for the highest doping concentration $\left(n_{2 D}=9 \times 10^{11} \mathrm{~cm}^{-2}\right)$, remaining below $3.6 \%$. Information extracted from the rocking curve is paralleled by the reciprocal space map (RSM) around asymmetric (224) reflections shown in Figure $2 b$ for the same sample. From the map, we obtain a clear picture of the strain conditions in the Ge and SiGe layers. By taking as a reference the dashed diagonal line, which corresponds to fully relaxed SiGe alloys, it is visually clear that both the $\mathrm{Ge}$ and the $\mathrm{Si}_{0.15} \mathrm{Ge}_{0.85}$ spots do not lie on this line, being, therefore, tensile strained in the growth plane with $\varepsilon_{/ /}=0.16 \%$ and $0.19 \%$, respectively. Thus, despite being plastically relaxed, the RG-VS is not fully relaxed due to the contribution of thermal strain, unavoidably arising from the difference in the thermal expansion coefficients of $\mathrm{Si}$ and $\mathrm{Ge}$, which builds up upon cooling down the samples to room temperature after the growth [31]. In addition, we note the perfect vertical alignment of all the SL fringes with respect to the $\mathrm{Si}_{0.15} \mathrm{Ge}_{0.85}$ buffer, indicating that the entire MQW stack is coherent with the in-plane lattice parameter of the underlying VS. 

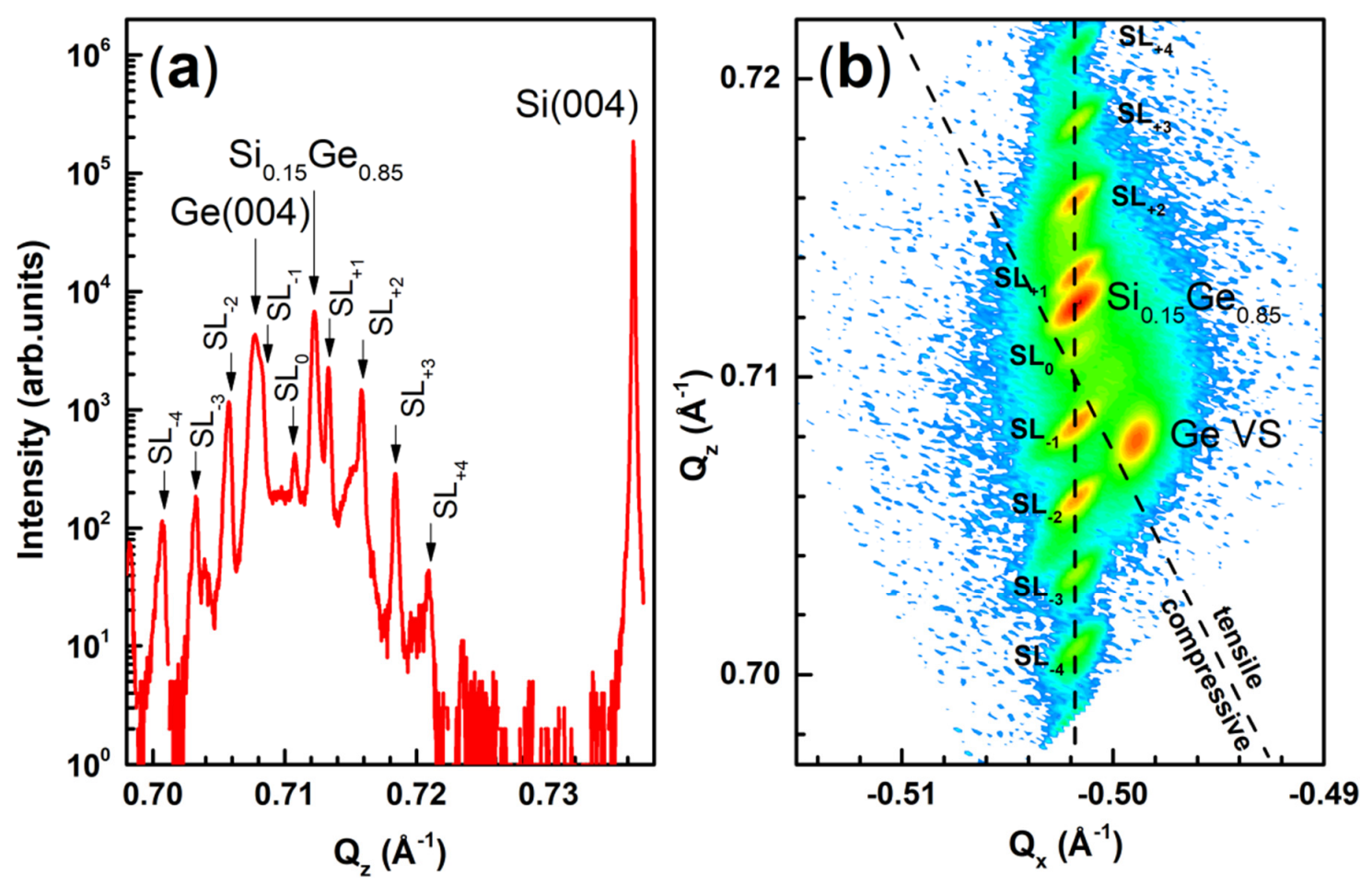

Figure 2. (a) XRD rocking curve around the (004) reflection and (b) XRD reciprocal space map (RSM) around the (224) reflections of the SiGe sample 2223. We labelled the peaks associated to the $\mathrm{Si}$ substrate, the Ge buffer and the thick $\mathrm{Si}_{0.15} \mathrm{Ge}_{0.85}$ alloy buffer of the VS, as well as different orders of the superlattice (SL) fringes.

We now focus on the SIMS calibration of the $N_{3 D}$ donor density in Ge films obtained by phosphine co-doping and its correlation to the actual carrier density $n_{2 D}$, measured by FTIR. To this end, we grew a set of Ge/SiGe MQWs (20 periods) where the $\mathrm{PH}_{3}$ partial pressure $p_{P H 3}$ was varied within a wide range of values. To be meaningful for $\mathrm{PH}_{3}-\mathrm{GeH}_{4}$ co-depositions, such values of pressure need to be normalized to the $\mathrm{GeH}_{4}$ flux $\phi_{\mathrm{GeH} 4}$, defining a reference parameter $P_{\mathrm{PH} 3}=p_{\mathrm{PH} 3} / \phi_{\mathrm{GeH} 4}$ in units of mTorr/sccm. This quantity is related to the measured $N_{3 D}$ in Figure 3a (red dots), showing that $n$-type doping of Ge is doable till the high $10^{19} \mathrm{~cm}^{-3}$ range where we observe a plateau in the donor density. Note that SIMS detection of $N_{3 D}<2 \times 10^{17} \mathrm{~cm}^{-3}$ is particularly challenging in the SiGe system due to the crosstalk of the ${ }^{31} \mathrm{P}^{-}$signal with hydrogenated $\mathrm{Si}$ species and, thus, data are sparser in this range. On the samples with a doping range measurable by FTIR and relevant for optically active MQW stacks, we compared donor detection by SIMS to the estimation of $n_{2 D} / t$ (blue squares) where the sheet carrier density is obtained from optical absorption measurements. We find a good match between the two datasets, with the doping density estimated by FTIR being only slightly lower than the SIMS values. This indicates that carriers resulting from donor ionization effectively populate the confined ground state at the $L$ point in the well. Or, in other words, the density of defects in our samples is low enough not to evidence, at the doping densities explored, a mismatch due to the compensation of the $p$-type background arising from electrically charged defects (i.e., dislocations). 

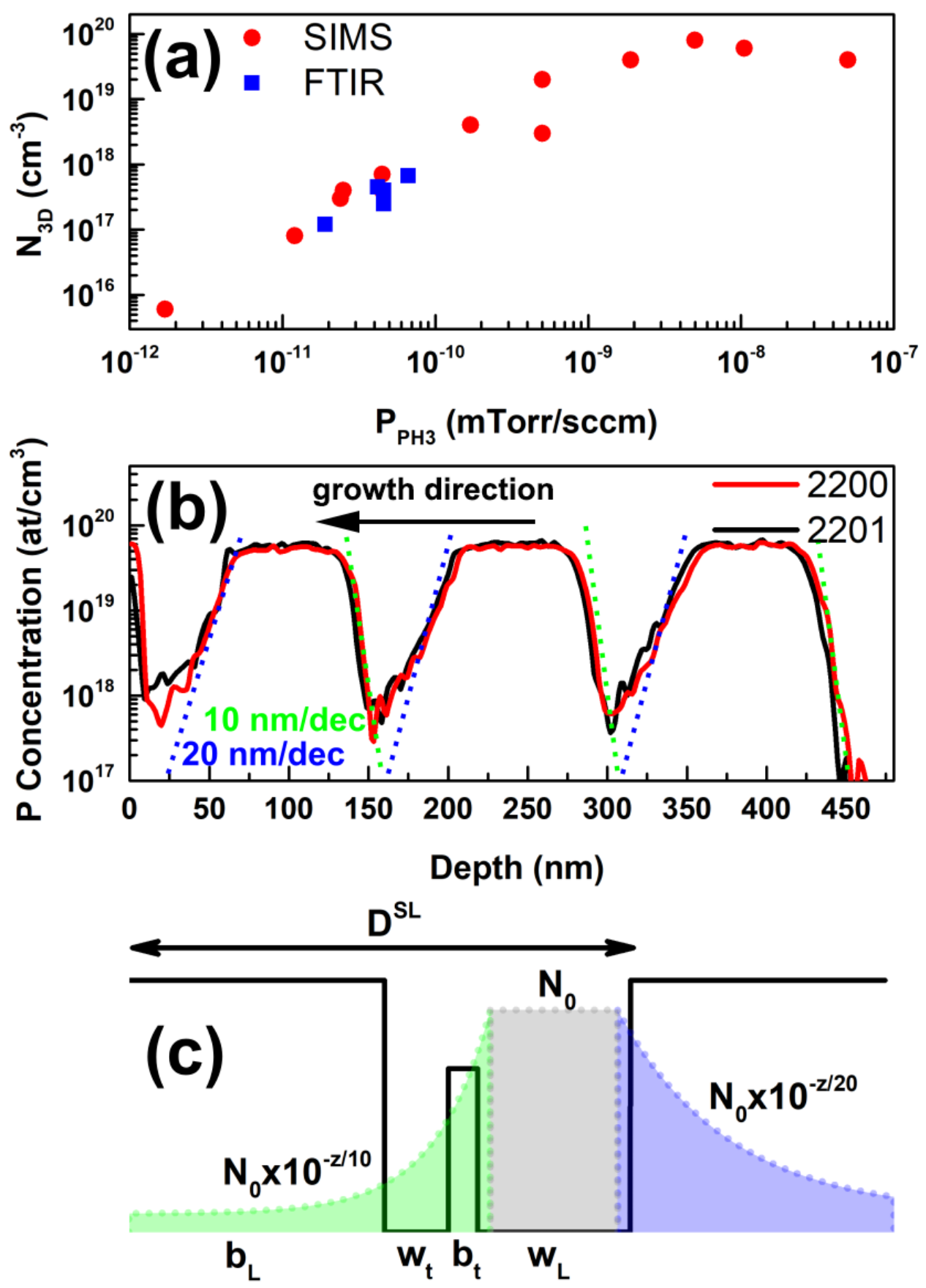

Growth direction z

Figure 3. (a) Correlation (red dots) between the $N_{3 D}$ concentration of $\mathrm{P}$ dopants obtained by secondary ion mass spectrometry (SIMS) and the growth parameter $P_{P H 3}$, i.e., the phosphine partial pressure normalized to the flux of the co-deposited germane gas. The same correlation plot (blue squares) but with the $N_{3 D}=n_{2 D} / t$ values obtained from the sheet carrier density $n_{2 D}$ measured by FTIR and the thickness $t$ of the doped layer. (b) Spatial profile of $\mathrm{P}$ dopants along the growth direction $z$ obtained by SIMS on two calibration samples featuring alternating doped and intrinsic layers. The diffusion tail of $\mathrm{P}$ atoms for the doped/undoped (undoped/doped) interfaces have been fitted to a mono-exponential decay function $N_{0} \times 10^{-z / d}$ displayed as green (blue) dots, where $d$ is the characteristic decay length and $N_{0}$ the $N_{3 D}$ density inside the doped layer. (c) Schematics of the ACQW module. The geometric parameters mentioned in the text are indicated. In the exponent of the decay function, $z$ is in nanometer. The donor diffusion in the leading and trailing directions is pictured. 
A realistic modelling of ISB optical absorption demands the accurate input not only the absolute value of the doping density, but also how the donors are spatially distributed along the growth direction. This is obtained through a spatially resolved mapping of the dopant $\mathrm{P}$ atoms by SIMS on calibration samples (Figure 3b). Following the direction of the growth (black arrow in Figure 3b), at the interfaces between the doped/undoped regions, we observed a leading edge-out diffusion of the donors with an exponentially decaying tail of $20 \mathrm{~nm} /$ decade (violet tail in Figure 3c). Diffusion in the trailing edge of the reversed undoped/doped interface (green tail) is narrower $(10 \mathrm{~nm} /$ decade), suggesting that the segregation of dopants occurring simultaneously as the growth proceeds is dominant over dopant diffusion at later stages of the growth. The obtained doping profile is schematically superimposed to the ACQW design in Figure 3c, showing that, even in the growth configuration where the doped well is grown at the end of the repeated module, a non-negligible density of donors is found inside the tunnel barrier layer. This significantly affects the energy position of the subband minima and, therefore, the optical absorption spectra discussed in the following subsections.

\subsection{Optical Absorption Measurements}

From the experimental absorption coefficient $\alpha_{2 D}(\omega)$ (shown in Figure $4 \mathrm{a}-\mathrm{d}$ ), we can extract both the linewidth and the energy position of ISB transitions for different design parameters. The corresponding theoretical spectra, calculated by the Schrödinger-Poisson solver, are reported in panels e-h. Focus first on samples 2218 and 2219 (Figure 4a,b,e,f): the two samples share the same geometrical parameters, but their doping concentrations differ by almost one order of magnitude (see Table 1 ). As detailed in the following, their common design $\left(w_{L} \cong 11.5 \mathrm{~nm}, b_{t}=2.3 \mathrm{~nm}\right)$ targets a significant interwell coupling and, as a result, the absorption spectra of both the samples at $T=10 \mathrm{~K}$ clearly show two absorption resonances, centered at $E_{01}^{a b s}$ and $E_{02}^{a b s}$, with similar oscillator strengths. These resonances correspond to transitions from the ground- $\left(L_{0}\right)$ to the first excited $\left(L_{1}\right)$ and the second excited $\left(L_{2}\right)$ subbands of the ACQW system, respectively. The appearance of both the resonances in 2218 and 2219 is the result of the breaking of the optical selection rules valid in symmetric systems (where a single ISB absorption peak is visible at low temperature) and demonstrates a significant overlapping of the envelope wavefunctions of the two wells for $b_{t}=2.3 \mathrm{~nm}$. This is confirmed by the simulations, which match very well the experimental spectra in terms of energy and spectral weight of the two resonances. In particular, we note that the energy position of the absorption resonances in 2218 is significantly blue shifted with respect to those in 2219 , due to the higher depolarization shift effect resulting from the heavier doping concentration of $2218\left(n_{2 D}=7.8 \times 10^{11} \mathrm{~cm}^{-2}\right.$, instead of 1.0 $\times 10^{11} \mathrm{~cm}^{-2}$ measured on 2219). Note that, in the two samples, the subband energy separations are, instead, practically overlapping, as evident by comparing Figure 4i,l. As a matter of fact, due to the depolarization shift, the absorption resonance does not occur at the subband energy separation $E_{i j}$ but rather at an energy given by $E_{i j}^{a b s}=\sqrt{1+2 e^{2} S_{i j}\left(N_{i}-N_{j}\right) \varepsilon^{-1} E_{i j}^{-1}}$, where $N_{i}$ is the $2 \mathrm{D}$ carrier population of the $i$-th subband, $\varepsilon$ is the material dielectric constant, $e$ the electron charge and $S_{i j}$ an effective length determined from the envelope wavefunction of the electron states involved [27]. Thus, at low temperature, where essentially only the ground state is populated, the magnitude of the depolarization shift is clearly proportional to the sheet carrier density $n_{2 D}$, which is consistent with our experimental observations. As mentioned in the Method section, the physical origin of the depolarization shift effect is related to the collective nature of the intersubband absorption, since the blueshift in the absorption spectrum is due to the charge carriers in the well partially screening the incident field [26].

By comparing the absorption spectra in Figure $4 \mathrm{a}$, e and 4b, f, corresponding to samples 2218 and 2219 which feature the same tunnel barrier thickness and very similar $w_{L}$ values but strongly differ in the doping concentration, we note that the lighter doped sample, 2219, shows smaller absorption linewidths. From a Lorentzian fit to the data, the $\Gamma_{1}\left(\Gamma_{2}\right)$ linewidths, evaluated as the half-width at half-maximum (HWHM) values of the absorption peaks at $E_{01}^{a b s}\left(E_{02}^{a b s}\right)$ is reduced from 7.5 (7.2) to 6 (5) $\mathrm{meV}$, when the doping is reduced from 7 to $1.0 \times 10^{11} \mathrm{~cm}^{-2}$. This is in line with previous observations in symmetric $n$-type Ge/SiGe MQWs where it was shown that, in this range of doping densities, ionized 
impurity scattering has a large impact on the absorption linewidths [32]. Having in mind the final target of realizing an emitter based on this material system, it is nonetheless interesting to note that, in a typical QCL design, the donors are spatially separated from the well where the optical transition occurs, since the latter is undoped, and thus ionized impurity scattering is expected to impact less on the emission linewidths. In addition, typical doping density in QCLs are also lower.
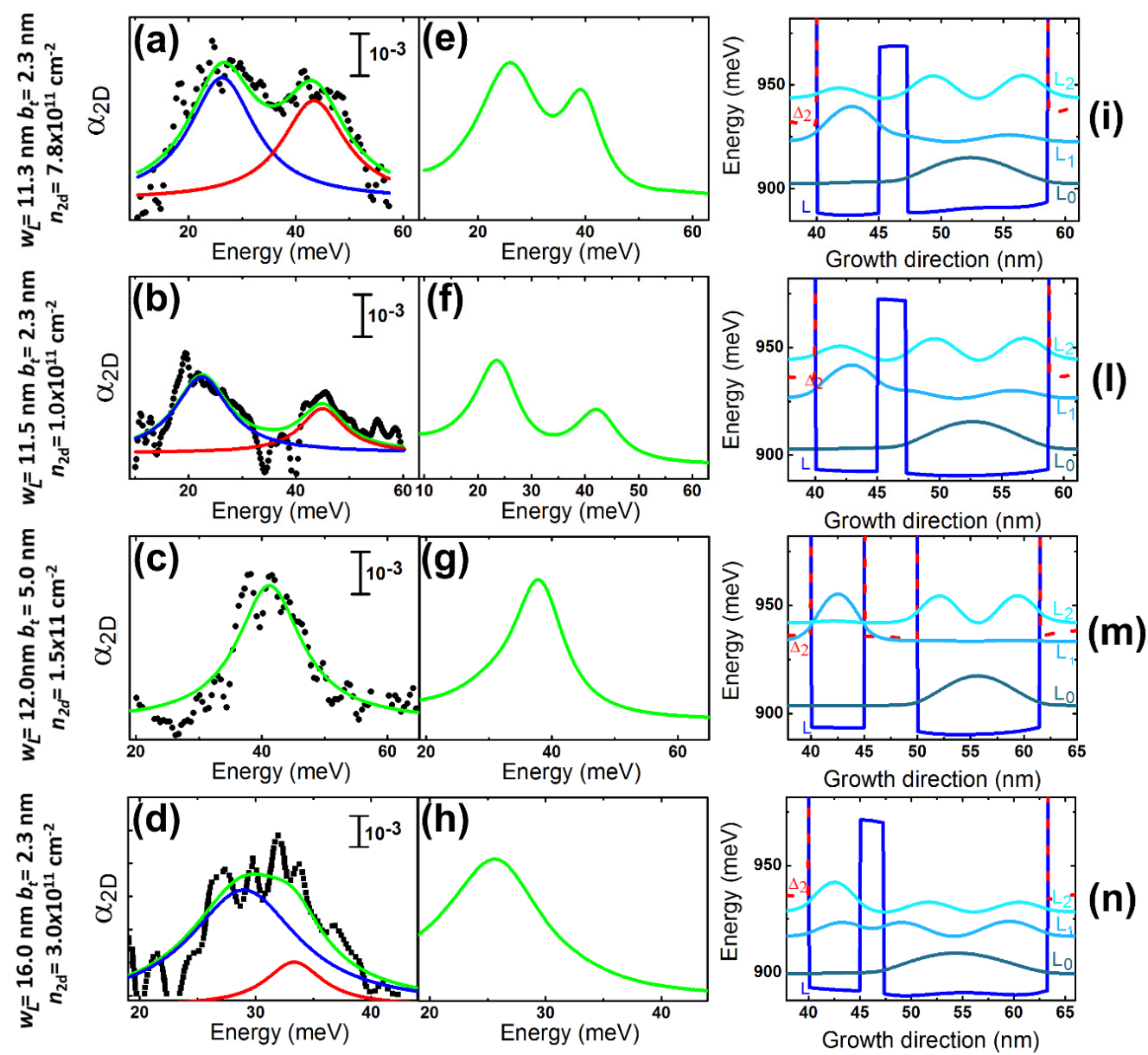

Figure 4. (a-d) Experimental intersubband (ISB) absorption measured by FTIR (black dots) with Lorentzian fits of the resonances centered at $E_{01}^{a b s}$ (blue curve) and $E_{02}^{a b s}$ (red curve). The green curve is the sum of the blue and red fit curves. (e-h) Corresponding calculated absorption spectra. (a,e) Sample 2218; (b,f) Sample 2219; (c,g) Sample 2224; (d,h) Sample 2267. (i-n) Calculated squared envelope wavefunctions for sample (i) 2218, (1) 2219, (m) 2224, (n) 2267.

We now correlate the geometrical parameters of the ACQW design to the features observed in ISB optical absorption spectra. The coupling of the excited electronic states of the two wells, which has a direct impact on the spectral features, is tunable by changing either the thickness of the tunnel barrier or the relative width of the two wells. In Figure 4, both approaches are presented with exemplificative experimental absorption spectra compared to their numerical counterparts. The effect of increasing the thickness of the tunnel barrier is clear when comparing the results obtained on samples 2219 and 2224 and shown in Figure $4 b, c$, respectively. These two samples have a similar well width, while $b_{t}$ changes from 2.3 (Sample 2219) to $5.0 \mathrm{~nm}$ (sample 2224). Hence, the $L_{1}$ envelope wavefunction in 2224 is strongly localized inside the thin well with a negligible penetration into the wide well, as shown by the calculated squared envelope wavefunctions in Figure $4 \mathrm{~m}$. The spatial overlapping of $L_{0}$ and $L_{1}$ is, therefore, vanishing and a single absorption resonance is observed in both the experimental and calculated spectra of 2224, since the $0 \rightarrow 1$ oscillator strength is negligible. Sample 2267 provides, instead, an exemplificative case of wavefunction engineering by tuning $w_{L}$. The related absorption 
experiments and simulations are shown in Figure 4d,h. With respect to samples 2218 and 2219, where $w_{L} \cong 11.5 \mathrm{~nm}$, here, the wide well width is increased to $16.0 \mathrm{~nm}$, while the tunnel barrier thickness is kept constant at $b_{t}=2.3 \mathrm{~nm}$. By comparing the calculated squared wavefunctions of 2267 (Figure $4 \mathrm{n}$ ) to those obtained for 2219 in Figure 41, we note that their shapes for the excited states differ significantly, in particular for $L_{2}$. As demonstrated by the simulation of the optical absorption coefficient $\alpha_{2 \mathrm{D}}(\omega)$ of 2267 displayed in Fig. 4h, the modified symmetry of the envelope wavefunctions drives a sizeable change in the spectral weights of the two ISB resonances, with the contribution centered at $E_{02}^{a b s}$ being markedly lower than in 2218 and 2219. More insights on such strong link between the geometric and electronic/optical properties of ACQW systems can be gained by analyzing in detail the results of the numerical model in the next section.

\section{Discussion}

Figure 5 a shows the energies of the three lowest-lying electron states $\left(E_{0}, E_{1}\right.$ and $\left.E_{2}\right)$ of the ACQW system as a function of $w_{L}$ and at a fixed $b_{t}=2.3 \mathrm{~nm}$ for $n_{2 D}=7.0 \times 10^{11} \mathrm{~cm}^{-2}$. We used the same color code as in the band diagrams of Figure 4. For an immediate comparison with the spectroscopy experiment, in Figure $5 b$, we report, as a blue (red) solid curve, the corresponding ISB transition energy $E_{01}=E_{1}-E_{0}\left(E_{02}=E_{2}-E_{0}\right)$ between the fundamental and the first (second) excited electron state. In the same panel, the values predicted for the optical resonance energy $E_{01}^{a b s}\left(E_{02}^{a b s}\right)$ are shown as a dotted blue (red) curve. The energy mismatch between ISB transitions and the corresponding optical resonances is due to the depolarization shift effect, which is fully accounted in the calculated spectra shown in Figure 4. The experimental optical absorption energies measured by FTIR on the samples featuring $b_{t}=2.3 \mathrm{~nm}$ and $n_{2 D} \cong 7 \times 10^{11} \mathrm{~cm}^{-2}$ are displayed by empty hexagonal markers, while the squares represent the optical absorption energies of the lower doped sample 2219. The experimental datapoints of the higher doped samples show a clear monotonic dependence with $w_{L}$, reproducing the behavior predicted by the model. As expected, the lower doped sample deviates from the hexagonal dataset due to its lower depolarization shift; the deviation is appreciable, in particular, for $E_{01}^{a b s}$, on which the depolarization correction is higher [25].

From the results of the calculations, we also note that the minimum separation between $E_{01}$ and $E_{02}$ is predicted to be around $w_{L}=14 \mathrm{~nm}$, which is the anticrossing point where $E_{1}=E_{2}$, in the limit of non-interacting wells (i.e., large tunneling barrier). The opening of a gap at the anticrossing reflects the hybridization between the states which, in the limit of uncoupled wells, represent the ground state in the narrow well and the first excited state in the wide well. From the inset of Figure $5 b$, where we show the oscillator strength $f_{01}\left(f_{02}\right)$ calculated for the $0->1(0->2)$ ISB transition as a function of $w_{L}$, the degree of state hybridization is evidently higher for $w_{L}<12 \mathrm{~nm}$. In this range, in fact, the two ISB transitions feature comparable oscillator strengths. By looking back at the spectroscopy data of Figure 4, this explains the similar spectral weights of the optical resonances for designs with $b_{t}=2.3 \mathrm{~nm}$ and $w_{L} \cong 11.5 \mathrm{~nm}$ (see panels a and $\mathrm{b}$ ). The significant state hybridization present in this configuration is visualized in the envelope wavefunctions shown in Figure 4i,l. From the same panels, we also note that, in the narrow well, the highest amplitude of the envelope wavefunctions is associated to $L_{1}$. Conversely, if we design the system with a $w_{L}$ value higher than the anticrossing condition as in 2267, where $w_{L}=16.0 \mathrm{~nm}$ and $b_{t}$ is still fixed at $2.3 \mathrm{~nm}$, we observe, instead, that the maximum amplitude inside the narrow well corresponds to the envelope wavefunction associated to $L_{2}$ (Figure $4 \mathrm{n}$ ). This can be explained considering that, when $w_{L}$ is lower (higher) than the anticrossing value $\left(w_{L} \cong 14 \mathrm{~nm}\right), L_{1}\left(L_{2}\right)$ represents the ground state of the narrow well in the limit of uncoupled wells [33]. As a consequence, when $w_{L}$ is increased, the $L_{2}$ amplitude in the wide doped well lowers significantly and, in turn, the oscillator strength $f_{02}$ is strongly suppressed [inset Figure $5 \mathrm{~b}$ ], matching the optical absorption experiments. 

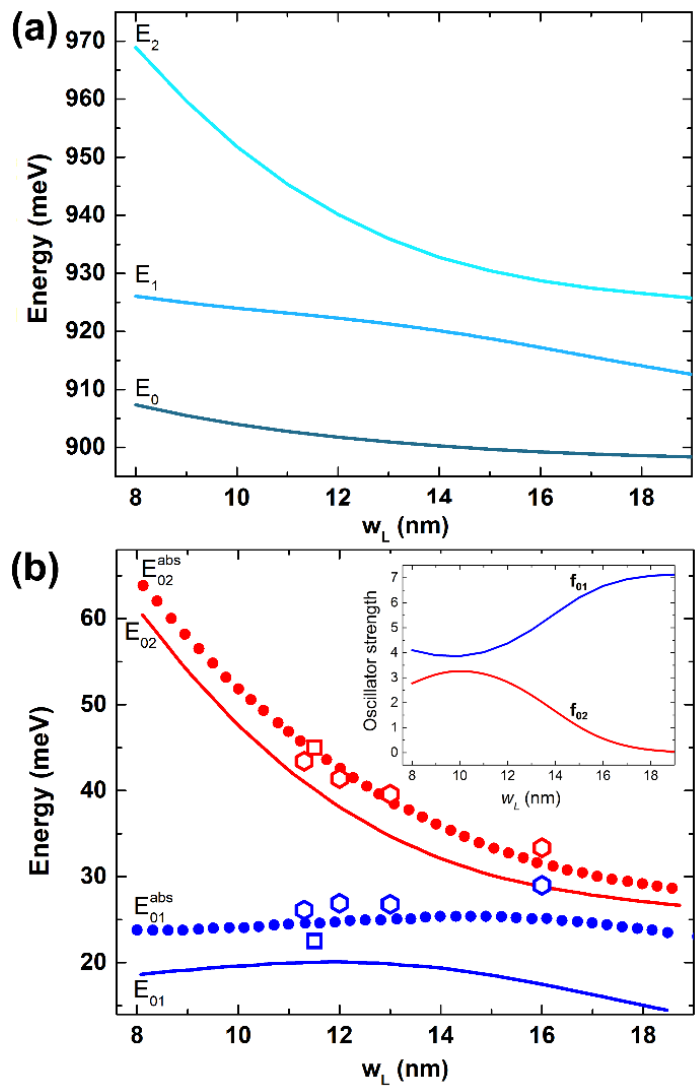

Figure 5. (a) Calculated energies of the fundamental $\left(E_{0}\right)$, first excited $\left(E_{1}\right)$ and second excited $\left(E_{2}\right)$ electron states for ACQW structures with a narrow well of $5 \mathrm{~nm}$ and a $\mathrm{Si}_{0.13} \mathrm{Ge}_{0.87}$ tunnel barrier $b_{t}=2.3 \mathrm{~nm}$ as a function of the wide well width $w_{L}$. (b) Corresponding energies of the $E_{01}$ and $E_{02}$ ISB transitions (continuous lines) and optical resonances (full dots). In the simulations, the sheet carrier density is $n_{2 D}=7 \times 10^{11} \mathrm{~cm}^{-2}$. The hexagonal (square) empty markers represent the experimental resonance energies obtained from FTIR spectra for samples with $n_{2 D} \cong 7 \times 10^{11} \mathrm{~cm}^{-2}$ $\left(n_{2 D}=1.0 \times 10^{11} \mathrm{~cm}^{-2}\right) . E_{01}^{a b s}\left(E_{02}^{a b s}\right)$ is shown in blue (red) color. In the inset, the calculated oscillator strength $f_{01}\left(f_{02}\right)$ for the $0->1(0->2)$ transition is displayed as a function of $w_{L}$ for the same design parameters as in the main panel.

\section{Conclusions}

By combining THz spectroscopy with numerical calculations of optical ISB absorption, we performed a detailed investigation of the dependence of quantum-confined electron states and ISB absorption resonances on the design of $n$-type Ge/SiGe ACQWs. The spectroscopic investigation was paralleled with an in-depth structural characterization obtained by merging STEM, XRD, and SIMS which showed excellent material quality and growth reproducibility.

Through the synergistic use of theory and experiments, we demonstrated the effective tuneability of the design of the energy of ISB transitions, as well as of the spatial overlap of the excited electronic wavefunctions. By varying the thickness of the tunneling barrier or the relative widths of the two coupled Ge wells, we extracted a rich ensemble of information from optical experiments, such as barrier heights, dipole matrix elements and level broadening due to scattering by ionized donor atoms. The very good agreement between the experimental and numerical calculations of optical ISB absorptions highlights the accurate estimation of the L-point conduction band offsets in the SiGe material system, for which we find a discontinuity of $117 \mathrm{meV}$ for $x_{\mathrm{Ge}}=0.81$ and of $80 \mathrm{meV}$ for $x_{\mathrm{Ge}}=0.87$, a range potentially suitable for the operation of a silicon-based THz QCL. 
Author Contributions: Conceptualization, L.P., M.O., M.V. and M.D.S.; methodology, M.D.S., M.V.; software, L.P., M.M., M.V.; validation, M.V. and M.D.S.; formal analysis, L.P.; investigation, L.P., M.M., C.C., L.B., M.O., M.Z., S.M., O.M., G.C., L.D.G., M.V. and M.D.S.; resources, L.P., G.C., L.D.G. and M.M.; data curation, L.P., M.M., C.C.; writing—original draft preparation, L.P., M.M.; writing—review and editing, M.D.S., L.G., C.C., L.B.; visualization, L.P., M.M.; supervision, M.D.S.,G.C.,M.O, M.V.; project administration, M.D.S., G.C.; funding acquisition, G.C., M.D.S. All authors have read and agreed to the published version of the manuscript.

Funding: This work is supported by the European Union research and innovation programme Horizon 2020 under grant no. 766719-FLASH project.

Acknowledgments: We acknowledge the technical support of the Lime laboratory of the Roma Tre University.

Conflicts of Interest: The authors declare no conflict of interest. The funders had no role in the design of the study; in the collection, analyses, or interpretation of data; in the writing of the manuscript, or in the decision to publish the results.

\section{References and Note}

1. Bastard, G. Wave Mechanics Applied to Semiconductor Heterostructures; Wiley: New York, NY, USA, 1992.

2. Fox, M.; Ispasoiu, R. Quantum Wells, Superlattices, and Band-Gap Engineering. In Springer Handbook of Electronic and Photonic Materials; Kasap, S., Capper, P., Eds.; Springer International Publishing: Cham, Switzerland, 2017; p. 1. [CrossRef]

3. Barnham, K.W.J.; Ballard, I.; Connolly, J.P.; Ekins-Daukes, N.J.; Kluftinger, B.G.; Nelson, J.; Rohr, C. Quantum well solar cells. Phys. E Low Dimens. Syst. Nanostructures 2002, 14, 27-36. [CrossRef]

4. Almosni, S.; Delamarre, A.; Jehl, Z.; Suchet, D.; Cojocaru, L.; Giteau, M.; Behaghel, B.; Julian, A.; Ibrahim, C.; Tatry, L.; et al. Material challenges for solar cells in the twenty-first century: Directions in emerging technologies. Sci. Technol. Adv. Mater. 2018, 19, 336-369. [CrossRef] [PubMed]

5. Welser, R.E.; Polly, S.J.; Kacharia, M.; Fedorenko, A.; Sood, A.K.; Hubbard, S.M. Design and Demonstration of High-Efficiency Quantum Well Solar Cells Employing Thin Strained Superlattices. Sci. Rep. 2019, 9, 13955. [CrossRef] [PubMed]

6. Köhler, R.; Tredicucci, A.; Beltram, F.; Beere, H.E.; Linfield, E.H.; Davies, A.G.; Ritchie, D.A.; Iotti, R.C.; Rossi, F. Terahertz semiconductor-heterostructure laser. Nature 2002, 417, 156-159. [CrossRef] [PubMed]

7. Williams, B.S. Terahertz quantum-cascade lasers. Nat. Photonics 2007, 1, 517-525. [CrossRef]

8. Vitiello, M.S.; Scalari, G.; Williams, B.; De Natale, P. Quantum cascade lasers: 20 years of challenges. Opt. Express 2015, 23, 5167-5182. [CrossRef]

9. Giorgioni, A.; Paleari, S.; Cecchi, S.; Vitiello, E.; Grilli, E.; Isella, G.; Jantsch, W.; Fanciulli, M.; Pezzoli, F. Strong confinement-induced engineering of the $\mathrm{g}$ factor and lifetime of conduction electron spins in Ge quantum wells. Nat. Commun. 2016, 7, 13886. [CrossRef]

10. Hendrickx, N.W.; Franke, D.P.; Sammak, A.; Scappucci, G.; Veldhorst, M. Fast two-qubit logic with holes in germanium. Nature 2020, 577, 487-491. [CrossRef]

11. Faist, J.; Capasso, F.; Sivco, D.L.; Sirtori, C.; Hutchinson, A.L.; Cho, A.Y. Quantum Cascade Laser. Science 1994, 264, 553. [CrossRef]

12. Gauthier-Lafaye, O.; Julien, F.H.; Cabaret, S.; Lourtioz, J.M.; Strasser, G.; Gornik, E.; Helm, M.; Bois, P. High-power GaAs/AlGaAs quantum fountain unipolar laser emitting at $14.5 \mu \mathrm{m}$ with $2.5 \%$ tunability. Appl. Phys. Lett. 1999, 74, 1537-1539. [CrossRef]

13. Paul, D.J. The progress towards terahertz quantum cascade lasers on silicon substrates. Laser Photonics Rev. 2010, 4, 610-632. [CrossRef]

14. Grange, T.; Stark, D.; Scalari, G.; Faist, J.; Persichetti, L.; Di Gaspare, L.; De Seta, M.; Ortolani, M.; Paul, D.J.; Capellini, G.; et al. Room temperature operation of n-type Ge/SiGe terahertz quantum cascade lasers predicted by non-equilibrium Green's functions. Appl. Phys. Lett. 2019, 114, 111102. [CrossRef]

15. Sabbagh, D.; Schmidt, J.; Winnerl, S.; Helm, M.; Di Gaspare, L.; De Seta, M.; Virgilio, M.; Ortolani, M. Electron Dynamics in Silicon-Germanium Terahertz Quantum Fountain Structures. ACS Photonics 2016, 3, 403-414. [CrossRef]

16. Ciano, C.; Virgilio, M.; Bagolini, L.; Baldassarre, L.; Pashkin, A.; Helm, M.; Montanari, M.; Persichetti, L.; Di Gaspare, L.; Capellini, G.; et al. Terahertz Absorption-Saturation and Emission from Electron-doped Germanium Quantum Wells. Opt. Express 2020, 28, 7245-7258. [CrossRef] 
17. Sun, G.; Cheng, H.H.; Menéndez, J.; Khurgin, J.B.; Soref, R.A. Strain-free Ge/GeSiSn quantum cascade lasers based on L-valley intersubband transitions. Appl. Phys. Lett. 2007, 90, 251105. [CrossRef]

18. Sun, G.; Soref, R.A.; Cheng, H.H. Design of a Si-based lattice-matched room-temperature GeSn/GeSiSn multi-quantum-well mid-infrared laser diode. Opt. Express 2010, 18, 19957-19965. [CrossRef]

19. Virgilio, M.; Ortolani, M.; Teich, M.; Winnerl, S.; Helm, M.; Sabbagh, D.; Capellini, G.; De Seta, M. Combined effect of electron and lattice temperatures on the long intersubband relaxation times of $\mathrm{Ge} / \mathrm{Si}_{\mathrm{x}} \mathrm{Ge}_{1-\mathrm{x}}$ quantum wells. Phys. Rev. B 2014, 89, 045311. [CrossRef]

20. Ciano, C.; Virgilio, M.; Bagolini, L.; Baldassarre, L.; Rossetti, A.; Pashkin, A.; Helm, M.; Montanari, M.; Persichetti, L.; Di Gaspare, L.; et al. Electron Population Dynamics in Optically Pumped Asymmetric Coupled Ge/SiGe Quantum Wells: Experiment and Models. Photonics 2019, 7, 2. [CrossRef]

21. Ciano, C.; Virgilio, M.; Montanari, M.; Persichetti, L.; Di Gaspare, L.; Ortolani, M.; Baldassarre, L.; Zoellner, M.H.; Skibitzki, O.; Scalari, G.; et al. Control of Electron-State Coupling in Asymmetric Ge/SiGe Quantum Wells. Phys. Rev. Appl. 2019, 11, 014003. [CrossRef]

22. Driscoll, K.; Paiella, R. Design of n-type silicon-based quantum cascade lasers for terahertz light emission. J. Appl. Phys. 2007, 102, 093103. [CrossRef]

23. Valavanis, A.; Dinh, T.V.; Lever, L.J.M.; Ikonić, Z.; Kelsall, R.W. Material configurations for \$n\$-type silicon-based terahertz quantum cascade lasers. Phys. Rev. B 2011, 83, 195321. [CrossRef]

24. Montanari, M.; Virgilio, M.; Manganelli, C.L.; Zaumseil, P.; Zoellner, M.H.; Hou, Y.; Schubert, M.A.; Persichetti, L.; Di Gaspare, L.; De Seta, M.; et al. Photoluminescence study of interband transitions in few-layer, pseudomorphic, and strain-unbalanced Ge/GeSi multiple quantum wells. Phys. Rev. B 2018, 98, 195310. [CrossRef]

25. De Seta, M.; Capellini, G.; Ortolani, M.; Virgilio, M.; Grosso, G.; Nicotra, G.; Zaumseil, P. Narrow intersubband transitions in n-type $\mathrm{Ge} / \mathrm{SiGe}$ multi-quantum wells: Control of the terahertz absorption energy trough the temperature dependent depolarization shift. Nanotechnol 2012, 23, 465708. [CrossRef] [PubMed]

26. Helm, M. The basic physics of intersubband transitions. In Intersubband Transition in Quantum Wells: Physics and Device Applications I. Semiconductors and Semimetals; Liu, H.C., Ed.; Academic: San Diego, CA, USA, 2000; pp. 1-99.

27. Busby, Y.; De Seta, M.; Capellini, G.; Evangelisti, F.; Ortolani, M.; Virgilio, M.; Grosso, G.; Pizzi, G.; Calvani, P.; Lupi, S.; et al. Near- and far-infrared absorption and electronic structure of Ge-SiGe multiple quantum wells. Phys. Rev. B 2010, 82, 205317. [CrossRef]

28. Tersoff, J. Dislocations and strain relief in compositionally graded layers. Appl. Phys. Lett. 1993, 62, $693-695$. [CrossRef]

29. Montalenti, F.; Rovaris, F.; Bergamaschini, R.; Miglio, L.; Salvalaglio, M.; Isella, G.; Isa, F.; Von Känel, H. Dislocation-Free SiGe/Si Heterostructures. Crystals 2018, 8, 257. [CrossRef]

30. Grange, T.; Mukherjee, S.; Capellini, G.; Montanari, M.; Persichetti, L.; Di Gaspare, L.; Birner, S.; Attiaoui, A.; Moutanabbir, O.; Virgilio, M.; et al. Atomic-scale insights into diffuse heterointerfaces: From three-dimensional roughness analysis to a generalized theory of interface scattering. arXiv 2020, arXiv:2002.00851.

31. Capellini, G.; De Seta, M.; Zaumseil, P.; Kozlowski, G.; Schroeder, T. High temperature x ray diffraction measurements on $\mathrm{Ge} / \mathrm{Si}(001)$ heterostructures: A study on the residual tensile strain. J. Appl. Phys. 2012, 111, 073518. [CrossRef]

32. Virgilio, M.; Sabbagh, D.; Ortolani, M.; Di Gaspare, L.; Capellini, G.; De Seta, M. Physical mechanisms of intersubband-absorption linewidth broadening in s-Ge/SiGe quantum wells. Phys. Rev. B 2014, 90, 155420. [CrossRef]

33. Among our samples, the lowest interwell interaction is obtained in 2224, which features the thickest tunnel barrier, and where, consistently with $w_{L}=12.0 \mathrm{~nm}$, we observe the strongest amplitude of $L_{1}$ in the narrow well, see Figure $4 \mathrm{~m}$.

(C) 2020 by the authors. Licensee MDPI, Basel, Switzerland. This article is an open access article distributed under the terms and conditions of the Creative Commons Attribution (CC BY) license (http://creativecommons.org/licenses/by/4.0/). 